\title{
P04.79. Navigating breast and cervical cancer screening services for Tongan women in Southern California
}

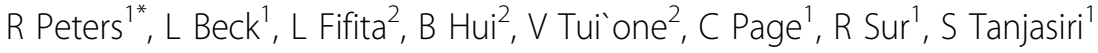 \\ From International Research Congress on Integrative Medicine and Health 2012 \\ Portland, Oregon, USA. 15-18 May 2012
}

\section{Purpose}

Patient Navigation (PN) has the potential to increase timely utilization of cancer prevention, early detection and treatment services among underserved populations. Few such navigators exist in community-based settings. Tongans in Southern California experience some of the largest cancer health disparities in the state. In addressing breast and cervical cancer, a pilot project was launched with the aim to increase cancer screening, treatment and support for Tongan American women ages 40 and above through cancer-related PN services.

\section{Methods}

The project consisted of assessment of patient-level barriers to screening, identifying and recruiting women for screening, and providing screening support and PN services to patients and their families. A mixed methods approach was used to analyze secondary data collected from Patient Intake and Tracking Forms. These forms collected data on demographics, health history, current cancer screenings and navigation services provided.

\section{Results}

A culturally and linguistically tailored breast and cervical cancer education and navigation project was established. Data showed major barriers to screening; $39.4 \%$ were uninsured, $35.1 \%$ had a high school diploma or less, $29.8 \%$ were non-English speaking, and $27.7 \%$ were undocumented. Results found that (1) Pap tests increased from $26.2 \%$ to $53.2 \%$ at the end of the project; (2) mammogram screenings increased from $29.1 \%$ to $62.0 \%$; (3) scheduling and coordination of screening appointments was the most frequent service (34\%), followed by reminders of upcoming screening appointments (18.1\%); and (4) the average number of contacts per patient was 2.36 suggesting multiple contacts are needed to overcome barriers to screening.

\section{Conclusion}

The pilot demonstrates screening needs of this underserved population, confirms lack of access as a primary barrier to screening, highlights the importance of PN in supporting women's screening, and underscores the importance of policies that support patient navigation in conjunction with the Patient Protection and Affordable Care Act, which expands health access to the medically underserved.

\section{Author details}

${ }^{1}$ California State University Fullerton, Fullerton, USA. ${ }^{2}$ Tongan Community Service Center/Special Service for Groups, Hawthorne, USA.

Published: 12 June 2012

doi:10.1186/1472-6882-12-S1-P349

Cite this article as: Peters et al:: P04.79. Navigating breast and cervical cancer screening services for Tongan women in Southern California. BMC Complementary and Alternative Medicine 2012 12(Suppl 1):P349.

${ }^{1}$ California State University Fullerton, Fullerton, USA

Full list of author information is available at the end of the article

(c) 2012 Peters et al; licensee BioMed Central Ltd. This is an Open Access article distributed under the terms of the Creative Commons Attribution License (http://creativecommons.org/licenses/by/2.0), which permits unrestricted use, distribution, and reproduction in any medium, provided the original work is properly cited. 